\title{
مفاتيح العزة: لوحة مفاتيح عربية لتسهيل كتابة وتعلم العربية في عصر المعلوماتية
}

Izza Keyboard: a new Arabic keyboard to facilitate Arabic learning and writing

\section{Abdelmalek Bouhadjera * أ. د. عبد المالك بوحجرة}

إن جودة التفاعل بين المستخدم العربي والحاسب الآلي عند إدخال النصوص العربية باستخدام لوحة المفاتيح تعبر عن مدى دقة وسهولة وتطور اللغة العربية تقتياً. ولكن الواقع المؤلم للعربية اليوم، المتمثل بعدم معالجة تلك المسألة بطريقة احترافية، يقلل من بن

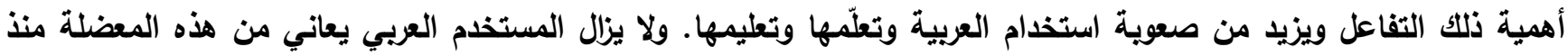
ظهور الآلة الراقنة (الكاتبة) إلى الآن، لأن الاقتراحات التي بنيت عليها تصاميم لوحات المفاتيح لم ترتكز في معظمها على دراسات منهجية ويحوث علمية لغوية، كما هو الحال في تصميم مفاتيح لغات الدول المتقدة. لذلك، اقترح تصميم جليد للوحة مفاتيح

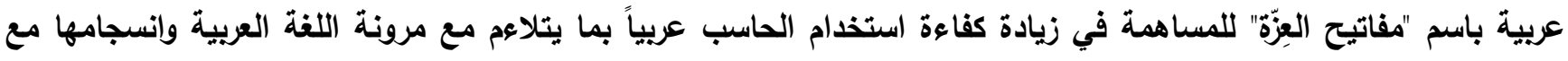
الحاسب. ومن أهم ميزات هذه اللوحة أنها تقلل من صعويات كتابة وتعلم اللغة العربية باستخدام الحاسب، وتعطي الهمزة استقلالية تامة باعتبارها حرفاً بذاته ممثلاً برمز و مفتاح خاص بها. باصنا بالإضافة إلى ذلك، هناك أزرار خاصة للحركات والثدّة مع المحافظة على توزيع الحروف الأساسي الموجود في لوحات المفاتيح الحالية.

\section{Abstract}

In this research, the focus is placed on the process of entering Arabic text via computer keyboards. The typing operation is a good indicator of the accuracy, ease of use and level of development of a particular language. However, this issue has not been treated in a professional manner in the Arab countries since the appearance of typewriters, due to the absence of real scientific research culture and technological development in Arab institutions. To mitigate this issue, a new Arabic keyboard, called Arabic Izza Keyboard, was designed to reflect a better picture of Arabic and to take advantage of its great flexibility and compatibility with computer use. A software application was also developed to reduce the obstacles in Arabic writing and learning, particularly by considering the Hamza as a single letter represented independently on a single key on the keyboard. The accents (Harakat and Shadda) are also given independent keys on the first keyboard level, without the need to press the shift key. Other improvements are implemented without affecting the current distribution and layout of most keys, making it easier and intuitive to write and deal with Arabic texts on computers.

كلمات مفتاحية: لوحة مفاتيح عربية، مفاتيح العزة، تعليم العربية، التفاعل بين الحاسب والإنسان، كتابة الأحرف

$$
\text { العربية، الكتابة العربية بحروف منفصلة، الكتابة العربية بحروف متصلة. }
$$

الأستاذ الدكتور عبد المالك بوحجرة، الجزائر، مر اسلة إلى: Prof Dr. Abdelmalek Bouhadjera, Algeria, Email: bouhadjeraa@yahoo.com 
تحنل اللغة العربية مكانة علمية ومعنوية مرموقة بين لغات الأمم، ويرجع ذلك بالدرجة الأولى إلى تاريخها الطويل

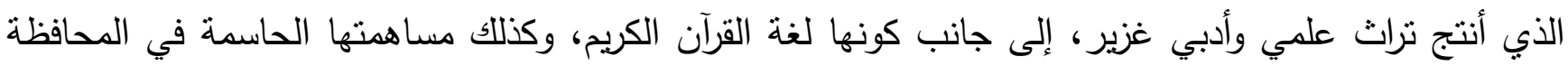

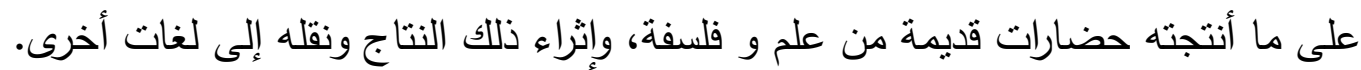

ولكن من الملاحظ اليوم أن واقع العرب والمسلمين لا يعكس بصفة عامة قوة اللغة العربية الحقيقية ومرونتها. حيث لا يشعر المرء بأن الثعوب و الدول التي تدعي و تتظاهر باستعمال العربية ترقى إلى مستوى لغتهم. إن المكانة

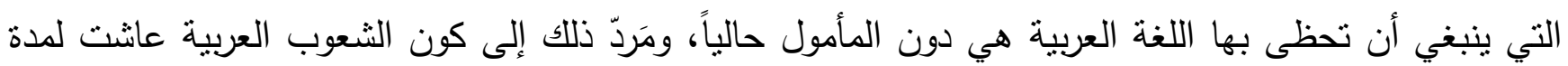
طويلة، و لا تزال، على هامش الحضارة الإنسانية. وما دامت لا تسيطر على العلم و التقنية فهي لا تدرك أهمية

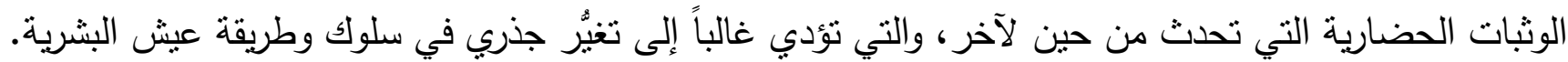
وكما حدث مع جل لغات العالم، فقد استفادت العربية بطريقة مباشرة وغير مباشرة من التقدم التقني الباهر في ترميز حروفها لتيسير استعمالها في ميدان المعلوماتية (فيما يتعلق بإدخال، وتخزين، ومعالجة، وإرسال وعرض المعلومات

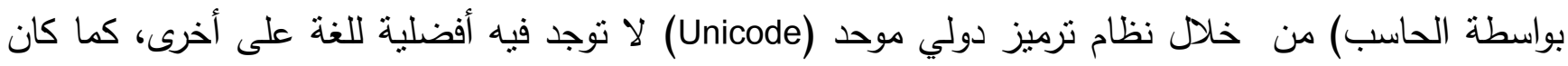
الأمر عند ظهور الحاسب (1). وباستعمال تقنية الخط المفتوح (Opentype)(2)، التي تعمل بانسجام مع نظام الترميز الموحد، وأنظمة تشغيل الحاسب المختلفة، يمكن للمصممين حالياً تصميم خطوط متتوعة لمختلف اللغات.

ويمكن اعتبار هذا التقدم التقني، في غياب تأثثر العرب، نِعمة ونِقمة في آن واحد على اللغة العربية. نعمة، لأن

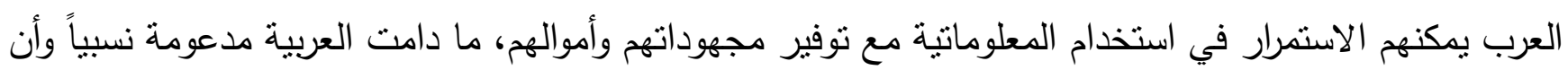

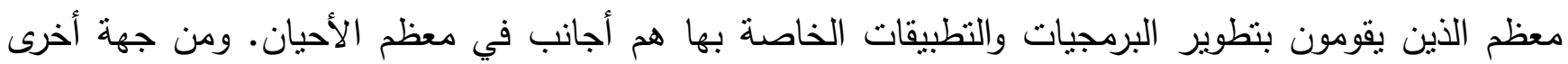
نقمة، لأن الإخفاقات التي حدثت في الماضي، وأدت إلى نأخر العربية في هذا العصر مقارنة مع لغات الدول

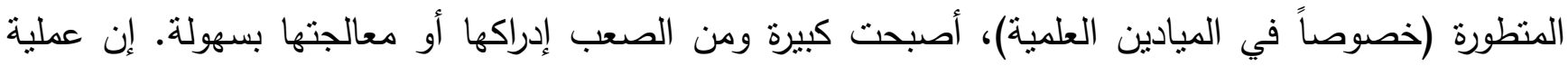
تطوير أب لغة وتكيفها مع الآلات الحديثة لا يمكن أن تتم إلا من طرف أهلها الذين يستعملونها و يمارسونها في إني

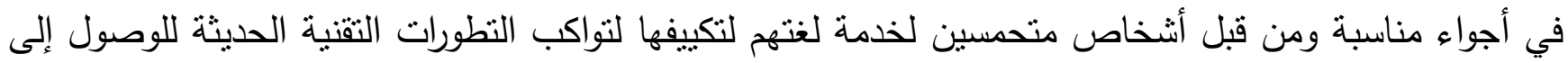
نتائج مقبولة في أفضل صورة إبداعية.

\section{2}

تم استتباط توزيع لوحة المفاتيح العربية من تصميم الآلة الراقنة (شكل 1)، الذي يعود إلى نهاية القرن التاسع عشر (3). حيث قام كثثر من صانعي أجهزة الحاسب بنطوير أزيد من عشرين تصميماً مختلفاً خلال سبعينيات وثمانينيات 


\section{A PATENTED ARABIC KEYBOARD}

The Arabc keyboard, as illustrated, was invented and patented some years ago by Mr. Selim S. Haddad, of Cairo, who represents the Monarch Typewriter for Egypt. Selim S. Haddad has sold a large number of Monarch Typewriters with this keyboard to the Egyptian Government and, we understand, Government officials in Cairo state that this is the most practical Arabic keyboard in use.

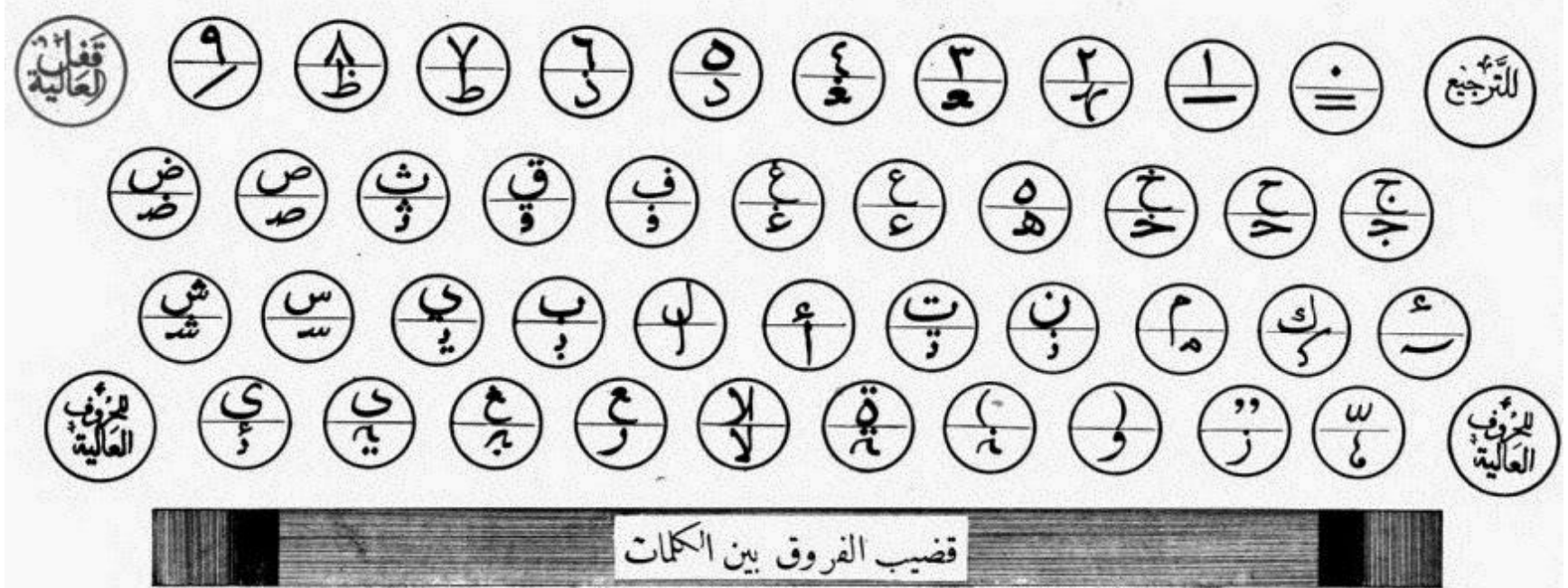

شكل (1) نمط توزع الحروف العربية على مفاتيح الآلة الز اقنّة (الآلة الكاتبة) من) Oztypewrite"، :مرجع 3)

القرن الماضي. واحتفظت كل التصاميم بتوزيع الحروف الموجود في الصف الثاني والثالث للآلة الراقنة (وهي الحروف: ا ب ت ث ج ح خ س ش ص ض ع غ ف ق ك ل م ى هـ)، ولكنها اختلفت في توزبع الحروف المتبقية على الصف الرابع (ؤ ة ز ظ و ى إ ذ أ د آ ىئ ر ط). ومن بين اللوحات المشهورة التي لا نزال في الاستعمال: لوحات ميكروسوفت Microsoft، ماكنتوش Macintosh و صخر (4). و كمحاولة للقضاء على فوضى التصاميم المتعددة، قامت المنظمة العربية للمواصفات والمقاييس بتطوير مواصفات لوحة مفاتيح عربية (ASMO 663) سنة 1987، مستمدة إلى حد كبير من تصميم الآلة الراقنة نفسها (شكل 2). ولكن لم يأخذ المصنعون بتلك المواصفات وطرحوا تصاميماً أخرى خاصة تتشابه جميعها تقريباً في المضمون.

\begin{tabular}{|c|c|c|c|c|c|c|c|c|c|c|c|c|c|c|c|c|}
\hline$=$ & $1^{!}$ & $2^{n}$ & $3^{\#}$ & $=$ & 4 & $5^{\frac{\circ}{\partial}}$ & $6^{\varepsilon}$ & 7 & 1 & $8^{\prime}$ & $9^{\prime}$ & 0 & & $=$ & $\wedge$ & \\
\hline & ض & ص & 3 & & تَ & ف & $\varepsilon$ & & $\varepsilon$ & 0 & $\dot{\tau}$ & & z & ]$^{]}$ & & \\
\hline & ش & 1 & $\dddot{2}$ & ىي & ب & J & ! & $1^{4}$ & ت & $\ddot{\Delta}$ & ى & $\prod_{2}^{1}$ & + & + & $*$ & آ \\
\hline & | ظ & ط & $j$ & & د & j & 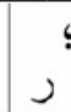 & & $=$ & ؤ & 6 & . & $>$ & ! & & \\
\hline
\end{tabular}

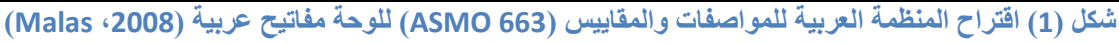


تُُنل الأبجدية العربية أصوات العربية بكفاءة منقطعة النظير، حيث تحنوي على عدد كبير من حروف الثد

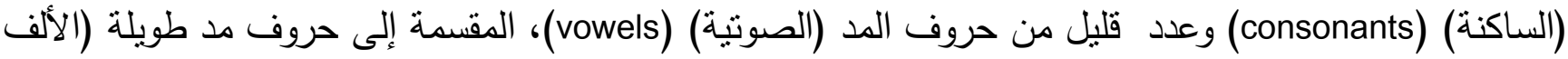
، والواو والياء) وحروف مد قصيرة (الفتحة، والضمة والكسرة)، التي تمثل بعلامات شكل و يمكن في معظم الأحبان الاستغناء عنها في الكتابة لأن العربية لغة موزونة، مما يعطي كتابة مختصرة للكلمات العربية، وهذا ما لا بمكن

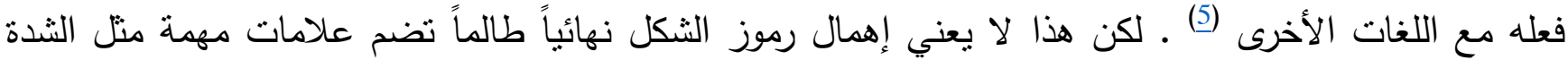
(التي ترمز إلى حرف مضاعف)، وهي ضرورية لإزالة اللبس عن بعض الكلمات. لذلك، يجب أن تكون علامات التشكيل متاحة بسهولة كباقي الحروف من خلال وضعها في المستوى الأول على لوحة الدفاتيح. إن تَصفّح سريع للأشكال 2، 3 و 4 يُظهر عمق المشكلة التي عانت ولا نزال نعاني منها اللغة العربية منذ زمن طويل، عندما نم الاستغناء عن الخطاطين في الكتابة. وييدو جلياً بأنه لم تتم الاستفادة من نقاط القوة التي تُميّز العربية وتكيفها مع مختلف الآلات.

\begin{tabular}{|c|c|c|c|c|c|c|c|c|c|c|c|c|c|c|}
\hline \begin{tabular}{cc|c}
$\sim$ & & $!$ \\
$\sim$ & $j$ & 1
\end{tabular} & $@$ & & $\begin{array}{l}\# \\
3\end{array}$ & 4 & $\begin{array}{l}\% \\
5\end{array}$ & $\hat{6}$ & $\begin{array}{l}8 \\
7\end{array}$ & 8 & & $\begin{array}{l}( \\
9\end{array}$ & $\begin{array}{l}\text { ) } \\
0\end{array}$ & $\begin{array}{l}+ \\
=\end{array}$ & $\stackrel{\leftarrow}{B a c}$ & Ekspace \\
\hline $\operatorname{Tab} \stackrel{\longleftrightarrow}{\longrightarrow}$ & & ص & ث & & & & & & 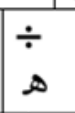 & $\stackrel{x}{\dot{x}}$ & $\begin{array}{l}\vdots \\
\tau\end{array}$ & $\begin{array}{l}> \\
7\end{array}$ & $\begin{array}{l}< \\
2\end{array}$ & 1 \\
\hline Caps Lock & شُ & " & & & ب & لا & أ & ت & ن & & ك) & b & Enter & \\
\hline Shift & & s & s & & 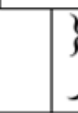 & & c & & ة & g' & j & ظُ & Shift & \\
\hline Ctrl & $\begin{array}{l}\text { Win } \\
\text { Key }\end{array}$ & Alt & & & & & & & & & Alt Gr & $\begin{array}{l}\text { Win } \\
\text { Key }\end{array}$ & Menu & Ctrl \\
\hline
\end{tabular}

شكل (2) توزع الحروف العربية على لوحة مفاتيح الحاسب الشخصي (بنظام تثشئل ويثلدز: Malas 2008) (IBM, PC)

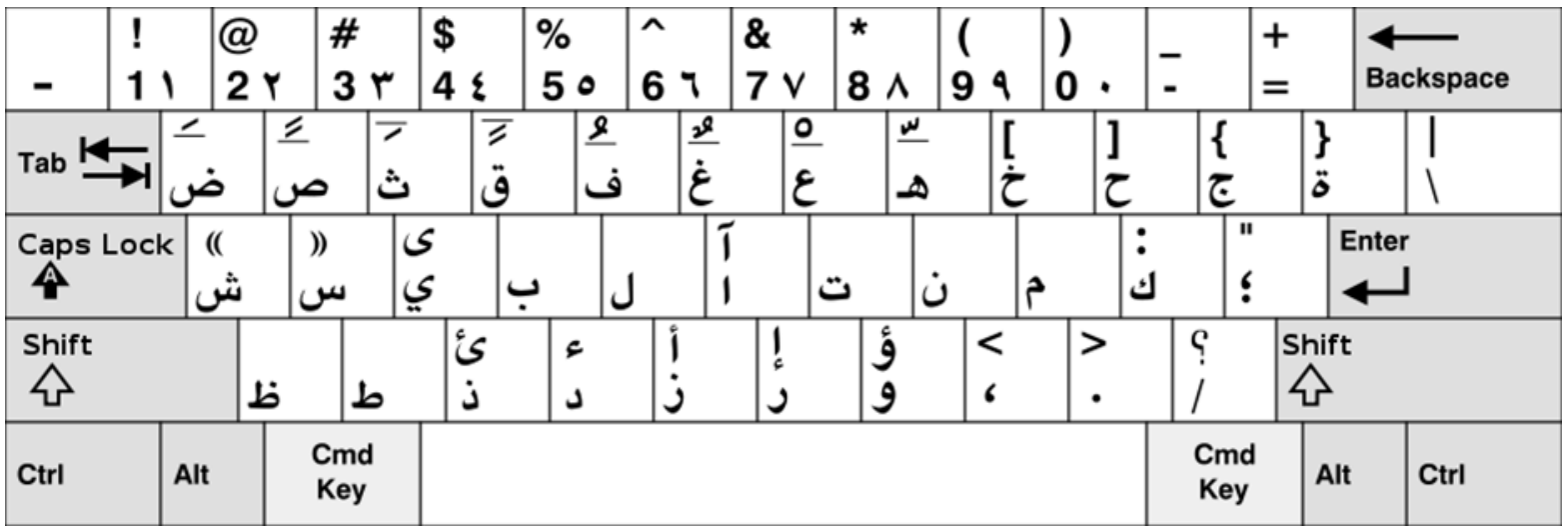

شكل (3) توزع الحروف العربية على لوحة مفاتيح نظام تشغيل ماكثتوش عربي (Mac Arabic) (2008) (Malas) 
فإلى جانب إهمال لوحة المفاتيح لعلامات التشكيل، التي ساهمت في تعقبد تدريس العربية للمبتدئين والأجانب،

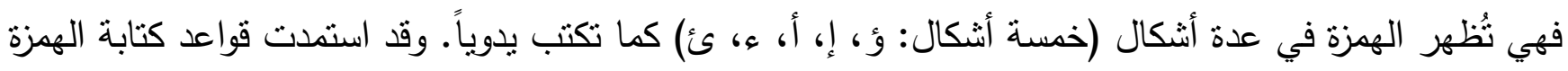
هذه من الخط المتصل، ولو تم الاهتمام بالخط المنفصل عندما ظهرت الطباعة لكان الأمر أسهل، لأنه من وجهة

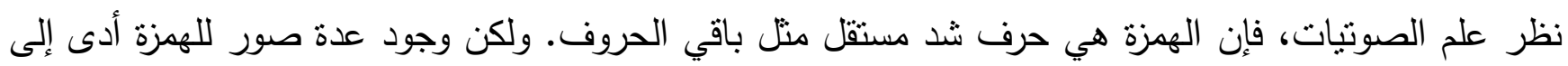

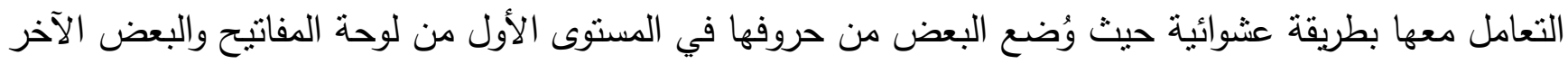

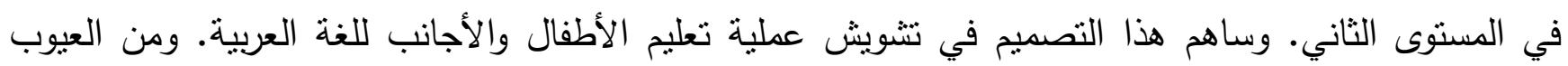

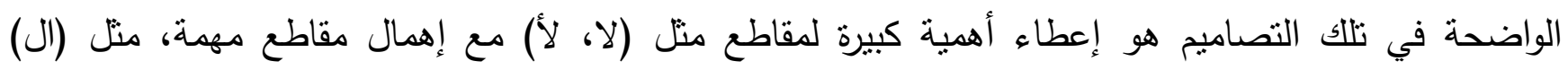
التعريف المتواجدة في أغلب الكلمات، وتوزيع الحروف والرموز المختلفة بطريقة فوضوية على المستوى الأول و الثاني بشكل لا يعكس نسبة استعمالها، وهذا ظاهر في كل تصاميم اللوحات الحالية.

\section{3}

نظرا لوجود تصاميح مختلفة من لوحات المفاتيح، فإنه من الضروري اقتراح لوحة مفاتيح موحدة تجمع كل البلدان

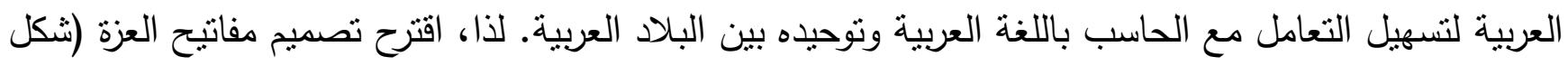

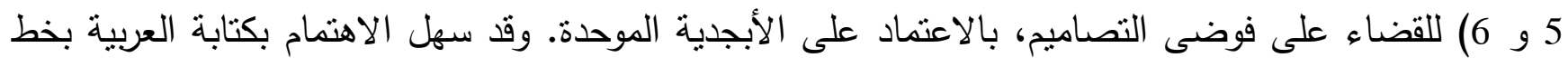

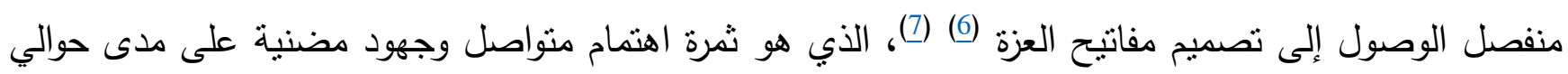

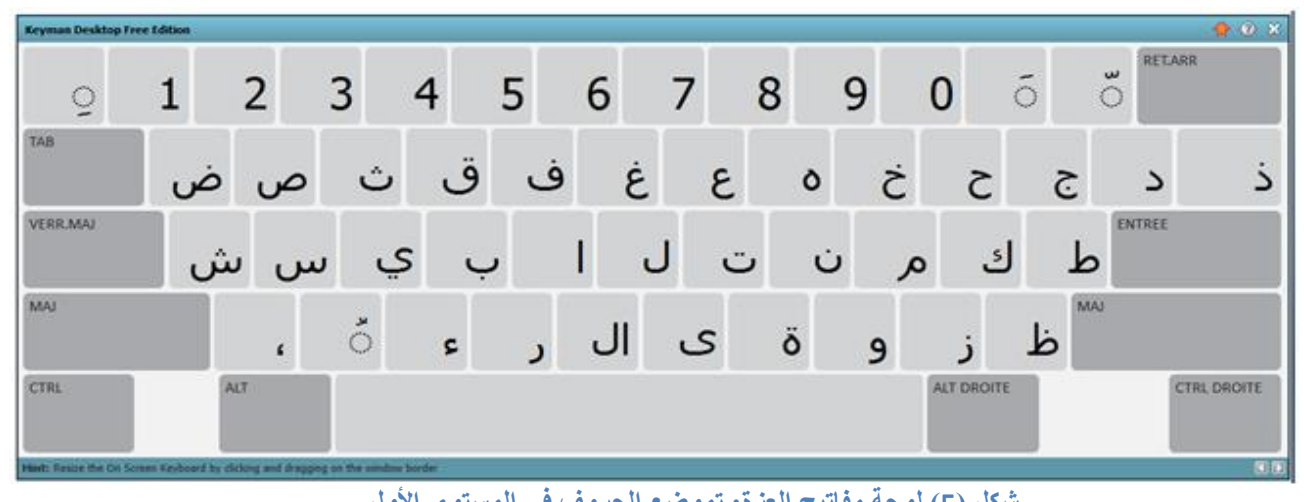
أربعين سنة.

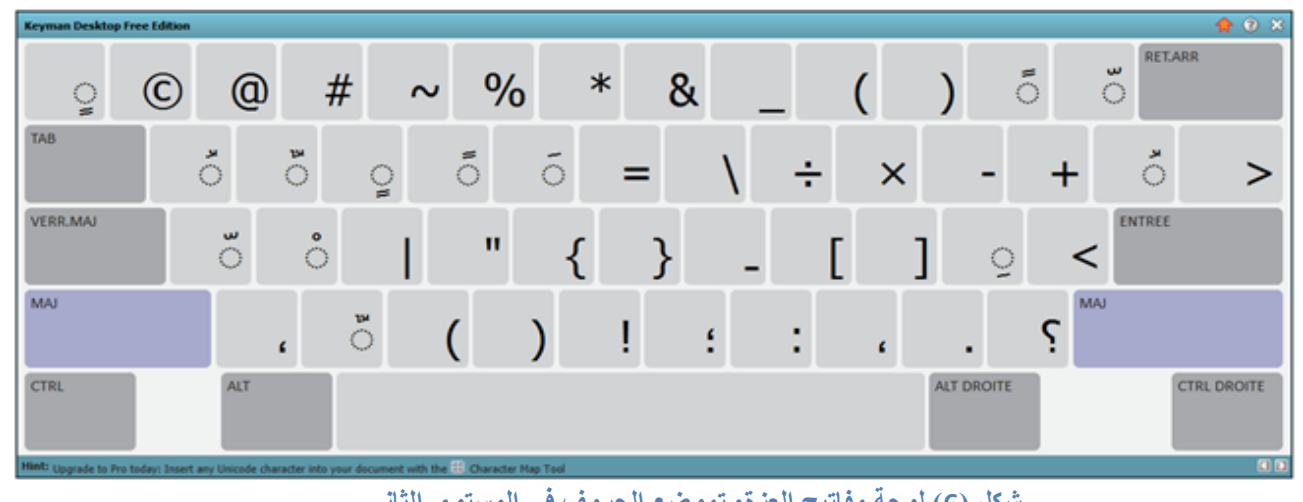

شكل (6) لوحة مفاتيح العزة: تموضع الحروف في المستوى الثاني 
هذا، وقد تطلّب استعمال الخط المنفصل تصميم خطوط جديدة دون اللجوء إلى توزيع جديد للوحة المفاتيح العربية. كما أن تمثيل الهمزة برمز واحد يؤدي إلى الاستغناء عن كثير من المفاتيح واستغلالها في تمثيل حركات أخرى. وبما أن الخطوط مبرمجة حسب لوحات المفاتيح المستعملة حالياً، فإن أي تغيير لتصاميم هذه المفاتيح ينطلب

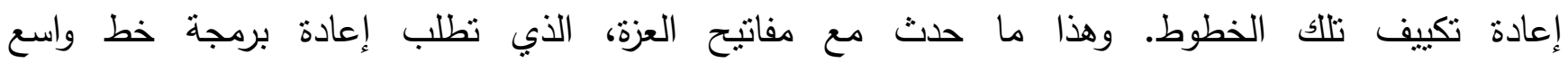
الانتشار(Simplified Arabic) للاستعمال مع التصميم الجديد، الذي تم إنجازه باستخدام برنامج مجاني من شركة ميكروسوفت، اسمه (MSKLC). ويمكن تحميل التطبيق الخاص بهذه اللوحة على أب جهاز حاسب لتجريبه واستخدامه في كتابة العربية (8).

\section{4) كتابة الهمزة في لوحة مفاتيح العزة}

يمكن كتابة الأشكال المختلفة للهمزة في لوحة مفاتيح العزة كما يلي:

- - كتنابة الهمزة فوق الألف (أ) يُضغط على حرف الألف (ا) مرتين متتاليتين.

- - كتابة الهمزة تحت الألف (إ) يُضغط على الهمزة (ع) مرتين متتاليتين.

- - كتابة الهمزة فوق الواو (ؤ) يُضغط على حرف الواو (و) مرتين متتاليتين.

- لكتابة الهمزة فوق النبرة (دُ) يُضغط على الألف المقصورة (ى) مرتين متتاليتين.

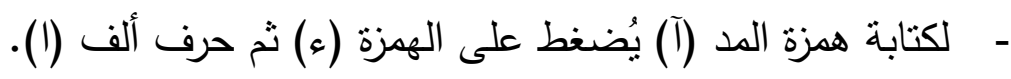

- - لكتابة الهمزة بمفردها الضغط على مفتاح الهمزة مرة واحدة فقط.

5) الميزات الأساسية لمفاتيح العزة

تتميز لوحة مفاتيح العزة بعدد من المواصفات التي يمكن تلخيصها بما يلي:

- تصميم مبسط مستمد من روح العربية يبرز في نفس الوقت جمالها و تناسقها على عكس اللوحات الحالية.

- - القضاء على مشكل كتابة الهمزة باستعمال زر واحد فقط.

- وجود مفاتيح مستقلة للحركات و الثدة على المستوى الأول.

- مفتاح مستقل لـ (ال) التعريف "الد".

- كتابة نصوص مشكّلة بسهولة و دقة دون الحاجة إلى استخدام المستوى الثاني باستعمال مفتاح "Shift". -

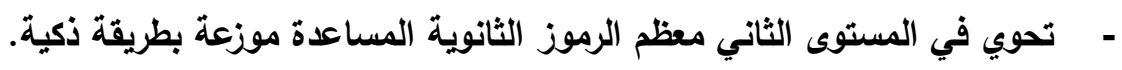

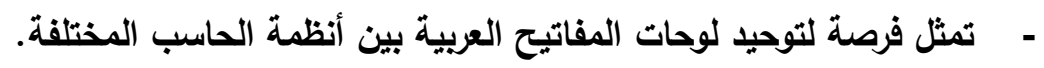
- تسهيل عملية إذخال النصوص العربية وتوفير الوقت والجها اللازم لذلك. 


\section{- - تقليل كثير من الأخطاء الثائعة عند استعمال لوحات المفاتيح الحالية. \\ - تغيير الفكرة المترسخة في أذهان كثير من الناس عن صعوية الاحطية اللغة العربية. \\ - تسهيل تعلم العربية للمبتدئين والأجانب من خلال الحاسب، وزيادة استعمالها في الإنترنت.}

جدير بالذكر أن تحريم الطباعة وعدم تقبُّها من قبل العرب والمسلمين لفترة طويلة، بعد مرور حوالي قرنين و نيف من ظهورها تقربياً (9)، كان أحد أسباب تلكؤ التعليم و تفشي الجهل و الأمية، وصعوبة اللحاق بالركب الحضاري فيما بعد. وهذا ما يستدعي الآن تطوير وتحديث وسائل تعلم اللغة العربية وتبسيطها والاهنمام بها علمياً وتقنياً بشكل متواصل، وتوفير أفضل وسائل الكتابة وتصاميمها، من خلال لوحات مفاتيح ذكية وسهلة تجعل تعلم اللغة العربية والكتابة بها أمراً ممتعاً لا منفراً.

\section{(6}

إن تبني لوحة مفاتيح العزة ودعمها من فبل الدول العربية سيساهم في تسهيل تعلم اللغة العربية وتسربع كتابتها على الحاسب، ويثجع المتعلمين على إدراك قيمتها وسهولتها واستعمالها مع الآلات الحديثة، ليس فقط في الأعمال والتعاملات التجارية وحسب، بل وفي كل ميادين الكتابة والتعليم والتدريس والتأليف والانتاج العلمي. ونحو تللك الغاية، فقد تم عرض الأبجدية الموحدة في المؤتمر الدولي الخامس للغة العربية بدبي، سنة 2016، تحت عنوان "الخط المعياري: سلاح ضد الجهل و التخلف"(10). كما أنه من الضروري إنشاء هيئة عربية جديدة للمواصفات، على غرار المنظمة العربية للمواصفات والمقاييس، التي تم حلّها سنة 1990 على الرغم من أهميتها، لتوحيد وتقوبة الجهود العربية لغوباً وتقنياً والاهتمام بالإعلام البصري و صناعة الخط العربي، التي لا تزال في مهدها وغالباً ما تثم خارج المنطقة العربية في مجملها.

\section{References}

1. Unicode. Available from: http://unicode.org/consortium/consort.html. Accessed 20 May 2018.

2. Microsoft Inc. Available from: https://docs.microsoft.com/en-us/typography/opentype/spec/. Accessed 20 May 2018.

3. Oztypewrite. Available from: https://oztypewriter.blogspot.com/2014/10/the-arabic-typewriterkeyboard-and.html. Accessed 20 May 2018.

4. Malas, T.M., S.S. Taifour, and G.A. Abandah, Toward optimal Arabic keyboard layout using genetic algorithm. Proc. 9th Int'I Middle Eastern Multiconf. on Simulation and Modeling (MESM 2008), 2008 : p. 50-54.

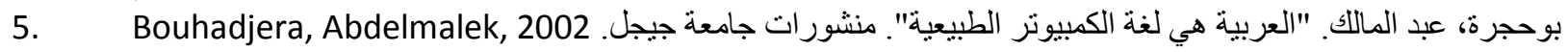

6. Bouhadjera, Abdelmalek. Wixsite, available from: https://bouhadjeraa.wixsite.com/mishkak. 
Arabic Science Archive (arabixiv.org) 2018

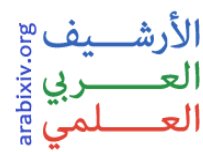

7. Available from: https://www.youtube.com/watch?v=f-OB7ABoyoQ. Accessed 20 May 2018.

8. Arabic Izza Keyboard, Available from:

https://www.tavultesoft.com/keyman/downloads/keyboards/details.php?KeyboardID=714\&FromKeyman=0.

9. Alwazzani, Hassan, Alarab Newspaper, 2016/07/23 Available from: https://alarab.co.uk/node/86492. Accessed 20 May 2018.

10. Bouhadjera, Abdelmalek. الخط المعياري سلاح ضد الجهل Available from: http://www.alarabiahconference.org/uploads/conference research-493512241-14592435682449.pdf. Accessed 19 May 2018.

DOI: http://dx.doi.org/10.17605/osf.io/p2y38 عبد المالك بوحجرة | مفاتيح العزة لتسهيل كتابة العربية وتعلمها | الأرشيف العربي العلمي 2018 